\title{
Management of tuberculous-contracted bladder with bilateral duplex collecting system: a case report with modified robotic urinary tract reconstructive surgery
}

\author{
Weijie Zhu ${ }^{1,2,3 \#}$, Zhihua Li $^{1,2,3 \#}$, Shubo Fan ${ }^{1,2,3}$, Xiang Wang ${ }^{1,2,3}$, Kunlin Yang ${ }^{1,2,3}$, Gengyan Xiong ${ }^{1,2,3}$, \\ Xuesong $\mathrm{Li}^{1,2,3}$, Liqun Zhou ${ }^{1,2,3}$ \\ ${ }^{1}$ Department of Urology, Peking University First Hospital, Beijing, China; ${ }^{2}$ Institute of Urology, Peking University, Beijing, China; ${ }^{3}$ National \\ Urological Cancer Centre, Beijing, China \\ \#These authors contributed equally to this work. \\ Correspondence to: Xuesong Li. Department of Urology, Peking University First Hospital, Beijing 100034, China; Institute of Urology, \\ Peking University, Beijing 100034, China; National Urological Cancer Center, No. 8 Xishiku St., Xicheng District, Beijing 100034, China. \\ Email: pineneedle@sina.com.
}

\begin{abstract}
Tuberculous bladder contracture with a bilateral duplicated collecting system is rare. According to anatomic variation, the surgical treatment strategy is highly individualized. We illustrate our robotic technique of urinary tract reconstruction. A 19-year-old girl with a history of pulmonary tuberculosis (TB) as a young child presented with a complaint of increasing frequency of micturition, nocturia, urgency, and urge incontinence starting at the age of 17 . Clinical and imaging examinations demonstrated tuberculous contracted bladder with a bilateral duplex collecting system. The patient underwent a robot-assisted Institute of Urology Peking University (IUPU) orthotopic ileal neobladder reconstruction. This is a modified urinary tract reconstructive method, including resection of the end of the duplex ureters and diseased contracted bladder with preservation of the proximal urethra and bladder neck, ileal harvesting and IUPU strategy to reconstruct an ileal neobladder, uretero-ileal anastomosis and neobladder-bladder neck anastomosis. The patient remained symptom-free without recurrence of TB and had improved renal function during the oneyear follow-up after surgery. Thus, our robot-assisted IUPU orthotopic ileal neobladder reconstruction method is an effective approach for this benign case. It can effectively increase bladder capacity, reduce intravesical pressure, and improve symptoms such as urination frequency and urgency.
\end{abstract}

Keywords: Tuberculous contracted bladder; orthotopic ileal neobladder; duplex collecting system; reconstructive surgery; case report

Submitted Jun 15, 2021. Accepted for publication Sep 01, 2021.

doi: 10.21037/tau-21-535

View this article at: https://dx.doi.org/10.21037/tau-21-535

\section{Introduction}

Genitourinary tuberculosis (GUTB), the second most common extra-thoracic tuberculosis (TB), occurs due to the metastatic spread of organisms through the bloodstream during an initial infection (1). The kidney is usually the primary organ involved in urinary $\mathrm{TB}$, while other parts of the urinary tract are affected by direct extension through urine. Chronic bladder TB is often secondary to renal TB accompanied by bilateral or unilateral renal damage, ureteral stricture, vesicoureteral reflux (VUR), or unilateral renal insufficiency. Insidious onset and difficulty in diagnosis lead to delayed treatment and may result in severe bladder fibrosis and scarring $(2,3)$.

The duplex collecting system is a common congenital malformation of the genitourinary system with an incidence of about $1-3 \%(4,5)$. According to ureteral morphology, duplicated kidneys can be incomplete or complete (6), unilateral or bilateral. Studies have reported that only $0.3 \%$ 


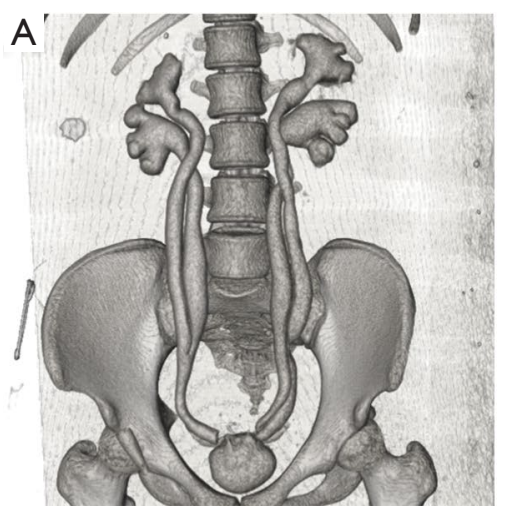

B

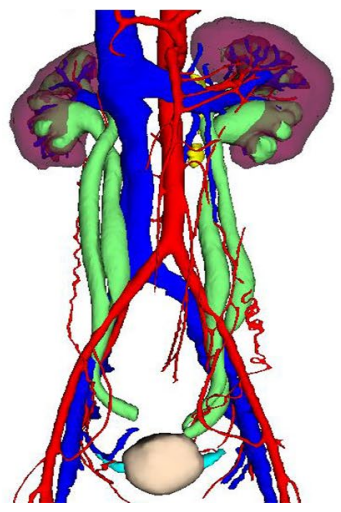

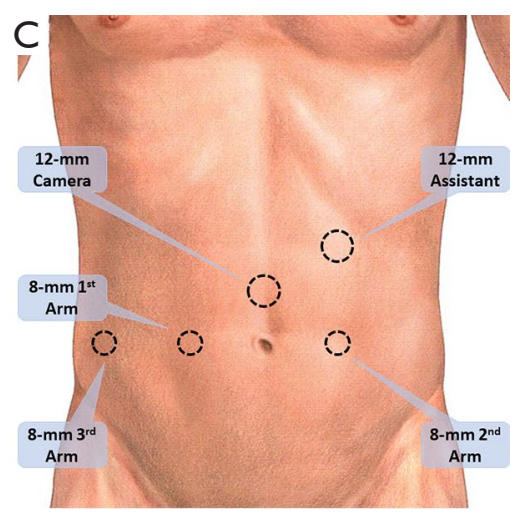

Figure 1 Preoperative evaluation three-dimensional (3D) computed tomography urography (CTU) and port placement. (A) Morphology of contracted bladder with bilateral duplex collecting system shown by traditional 3D CTU; (B) morphology of contracted bladder with bilateral duplex collecting system shown by 3D virtualization technology of CTU; (C) port placement of robot-assisted Institute of Urology Peking University (IUPU) orthotopic ileal neobladder reconstruction.

of patients have bilateral duplicated kidneys by excretory urography (7). A complete duplex collecting system is usually associated with other congenital anomalies, such as ectopic ureteric insertion, ureterocele, and VUR (8).

Here we present a case of a tuberculous contracted bladder with a bilateral duplex collecting system and our technique of robotic Institute of Urology Peking University (IUPU) orthotopic ileal neobladder reconstruction. We present the following case in accordance with the CARE reporting checklist (available at https://dx.doi.org/10.21037/ tau-21-535).

\section{Case presentation}

A 19-year-old girl with a history of pulmonary TB as a young child presented with a complaint of increasing frequency of micturition, nocturia, urgency, and urge incontinence starting at the age of 17 , occasionally accompanied by left-sided flank pain and fever. She was subsequently had a urine smear and a culture for acidfast bacilli, which was negative. She underwent a urine metagenomics test for mycobacterium TB, which was positive. She was then put on anti-tubercular therapy (ATT) for 6 months (Isoniazid, Rifampin, Pyrazinamide and Ethambutol for the first 2 months, then Isoniazid and Rifampin for the last 4 months).

After ATT, the patient continued to have symptoms to a lesser extent, but had bilateral flank pain. Renal function was normal, with a serum creatinine of $1.41 \mathrm{mg} / \mathrm{dL}$ (normal range, $0.498-1.505 \mathrm{mg} / \mathrm{dL}$ ). She underwent an abdominal ultrasound and computed tomography urography
(CTU) with three-dimensional (3D) virtualization technology, which showed a very-low capacity $(30 \mathrm{~mL})$ urinary bladder and duplex collecting system with mild hydroureteronephrosis (Figure $1 A, 1 B$ ). The right-side double ureters converged on the pelvic segment, presenting a "Y" shape with ectopic implantation to the bladder fundus. On the left side, the double ureters abnormally went directly into the top wall of the bladder. We performed a urodynamic study and cystourethrography and presented grade I VUR on the right side and grade IV VUR on the left. A $99 \mathrm{mTc}$-DTPA diuretic renal scan showed that the drainage of the bilateral collecting system was not impaired and the glomerular filtration rate of the right kidney was $37 \mathrm{~mL} / \mathrm{min}$, compared to $43 \mathrm{~mL} / \mathrm{min}$ on the left kidney (normal arrange in total: $\geq 68 \mathrm{~mL} / \mathrm{min}$, normal arrange of one side: $\geq 34 \mathrm{~mL} / \mathrm{min}$ ).

Surgical therapy was indicated for this patient, considering the symptoms, compromised split renal function, and the patient's willingness. All procedures performed in this study were in accordance with the Declaration of Helsinki (as revised in 2013) and approved by the Ethics Committee of Peking University First Hospital (approval number: 2020-283). Written informed consent was obtained from the patient for publication of this case report and accompanying images. A copy of the written consent is available for review by the editorial office of this journal.

\section{Surgical technique}

The patient was placed in an extended lithotomy 

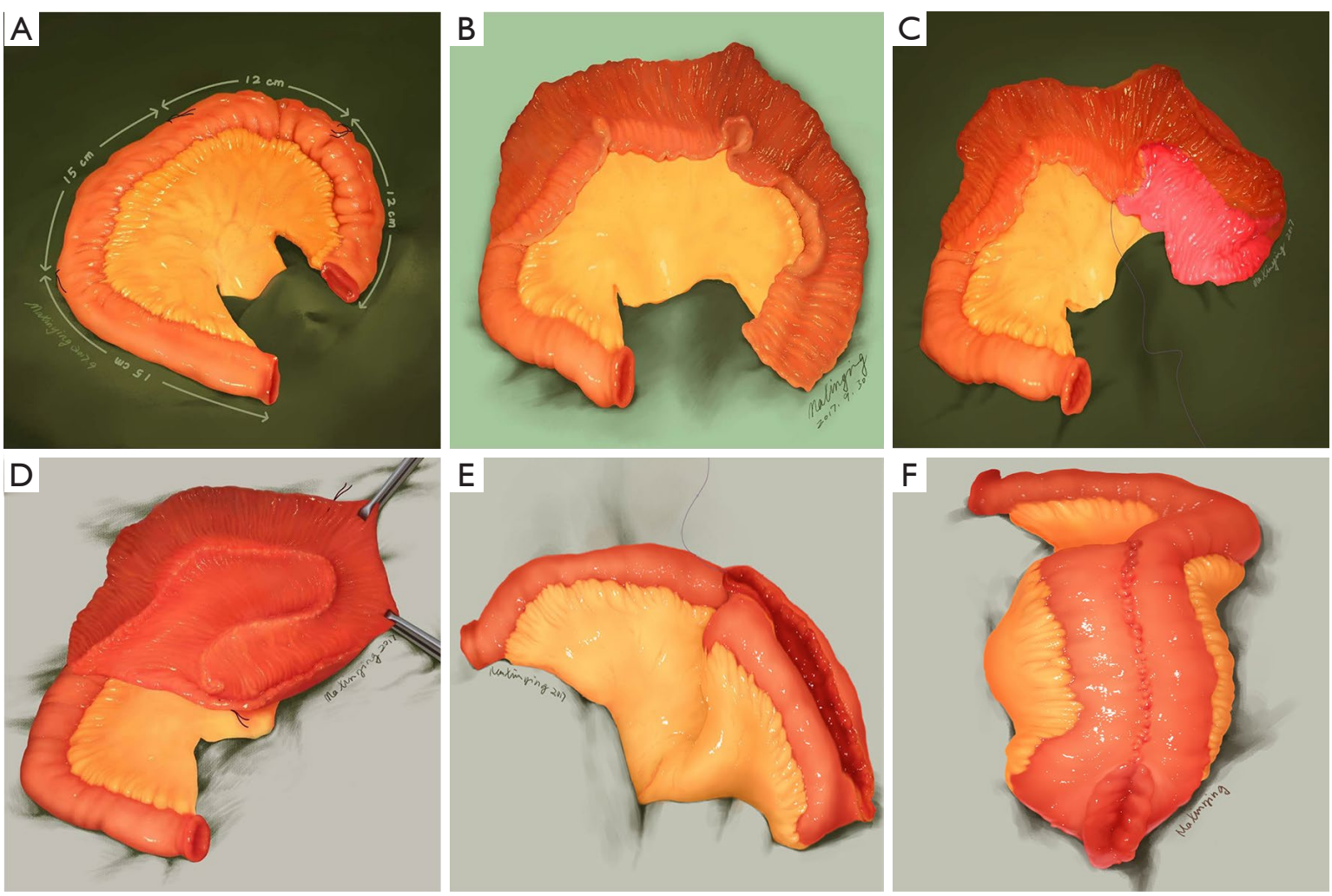

Figure 2 Schematic diagram of the procedures of Institute of Urology Peking University (IUPU) orthotopic ileal neobladder reconstruction. Reused with permission from reference (9). (A) Ileal segment to reconstruct the orthotopic neobladder; (B) detubularization of the ileal segment along the antimesenteric border; (C) first fold of the distal ileal segment; (D) second fold of the distal ileal segment to form the posterior wall of the neobladder; (E) the ileal pouch with the opening of anterior wall of the neobladder; (F) completion of the IUPU orthotopic ileal neobladder reconstruction.

Trendelenburg position with adequate padding of the pressure points. A Veress needle was used to achieve pneumoperitoneum. The $12-\mathrm{mm}$ port for the camera was placed $3 \mathrm{~cm}$ above the umbilicus first. All other ports were introduced under direct vision after the abdominal cavity was examined. Two $8-\mathrm{mm}$ robotic ports were placed $8-10 \mathrm{~cm}$ lateral to the umbilicus. Another $8-\mathrm{mm}$ robotic port was placed $8-10 \mathrm{~cm}$ lateral to the right robotic port. Another $12-\mathrm{mm}$ port was placed $8-10 \mathrm{~cm}$ above the left robot port as an assistant port (Figure 1C).

The diseased bladder and bilateral ureteral orifice were excised while preserving the proximal urethra and bladder neck. The ileum was removed from the abdominal cavity through a $5-\mathrm{cm}$ midline incision. We selected an ileum segment about $25 \mathrm{~cm}$ proximal to the ileocecal valve. The ileum segment was measured by calibrated drainage, and a $54-\mathrm{cm}$ segment was isolated for the neobladder by resecting the ileum and its adjacent mesentery. We marked the ileum segment using sutures at $12 \mathrm{~cm}$ (point of the first fold), $24 \mathrm{~cm}$ (point of the second fold), and $39 \mathrm{~cm}$ (point of the beginning of the afferent limb). The distal ileum was $39 \mathrm{~cm}$ in length for the pouch and the proximal ileum was $15 \mathrm{~cm}$ in length using the afferent limb (Figure 2A) (9). The extracorporeal functional side-to-side anastomosis was used to reconstruct intestinal continuity. The $39-\mathrm{cm}$ ileum segment was detubularized along the antimesenteric border (Figure 2B) (9). The distal 12-cm ileum segment was folded. The edges of the two neighboring $12-\mathrm{cm}$ ileal segments were connected with 3-0 absorbable sutures (Figure 2C) (9). The other distal 12-cm edge of the ileum was folded and aligned with the adjacent edge of the $15-\mathrm{cm}$ ileal segment (Figure 2D) (9). This is how the parallel free edges of the $12-\mathrm{cm}$ ileum and $15-\mathrm{cm}$ ileum were generated (Figure 2E) (9). The two free edges were sutured together, and an opening was left for anastomosing with the bladder neck (Figure 2F) (9). 

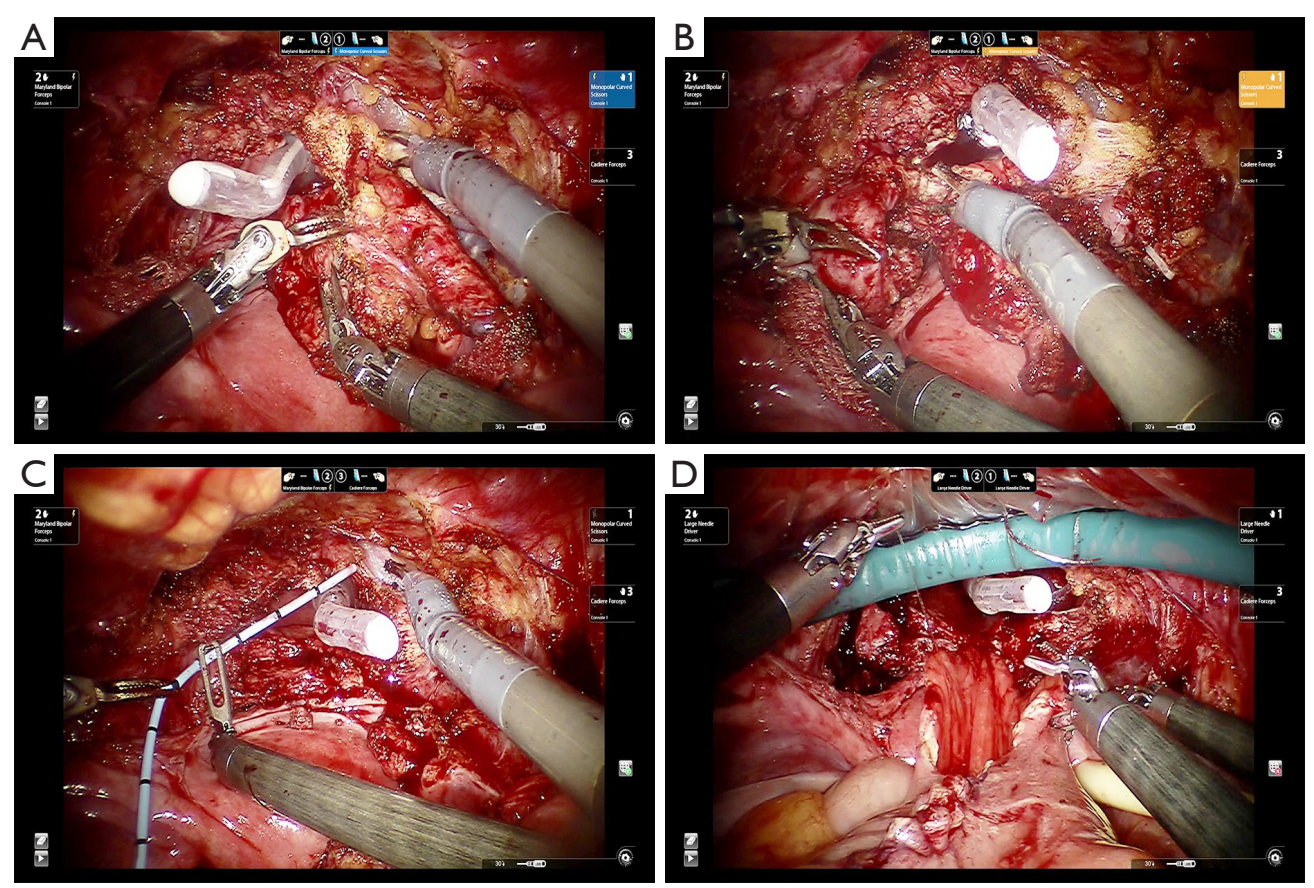

Figure 3 Intraoperative image of the procedures of Institute of Urology Peking University (IUPU) orthotopic ileal neobladder reconstruction. (A) Identification of the right ureter and resection of left ureteral orifice; (B) identification of the left ureter and resection of left ureteral orifice; (C) resection of the diseased bladder and measurement of the diameter of incision; (D) anastomosis between the bladder neck and the small opening of the neobladder.

One single-J stent of size $6 \mathrm{~F}$ was inserted into each of the four ureters. The left two distal ureters were longitudinally split at $2.5 \mathrm{~cm}$. The two ureters were then anastomosed into one $2.5-\mathrm{cm}$ combined ureter at the distal end. End-to-end anastomosis between the afferent limb and the combined ureter was performed in a running fashion with an absorbable Vicryl of 4-0 size. The right duplicated ureters merged into one at the distal end, and end-to-side anastomosis was made with the afferent limb. Neobladder cystostomy was performed and four single-J stents were fixed to a stoma catheter with non-absorbable sutures so they could be removed about 4 weeks after surgery. After re-docking, end-to-end anastomosis between the bladder neck and the small opening of the neobladder was performed using 2-0 Q sutures in a running method. After the neobladder was tested by irrigation to ensure that the closure was watertight, a three-way urinary catheter was inserted. Then, the pelvic drainage tube was placed at the left lateral port site and the surgical ports and incision were closed. Surgical robot-assisted procedures are shown in Figure 3.

\section{Results}

Pathological examination revealed chronic inflammation with squamous epithelium and granulation tissue of the bladder mucosa. The patient was treated with ATT for 6 months to avoid recurrence (Isoniazid, Rifampin, Pyrazinamide and Ethambutol for the first 2 months, then Isoniazid and Rifampin for the last 4 months). During the recovery period, irrigation of the neobladder began on the day after surgery in case of mucous congestion (about $100 \mathrm{~mL}$ saline every two hours). The urinary catheter was extracted about 3 weeks postoperatively after cystography (Figure $4 A$ ) and a cystostomy tube combined with 4 single-J stents was removed after a 1 -week urination training (Figure $4 B$ ). In case of metabolic acidosis and hypovolemia, the patient should drink 2-3 liters of water per day. The patient remained symptom-free without TB recurrence and had improved renal function during the 1-year follow-up.

According to the Clavien-Dindo classification system, no severe complications (grade $\geq 3$ ) occurred (10). The patient had mild metabolic acidosis that was treated conservatively. 

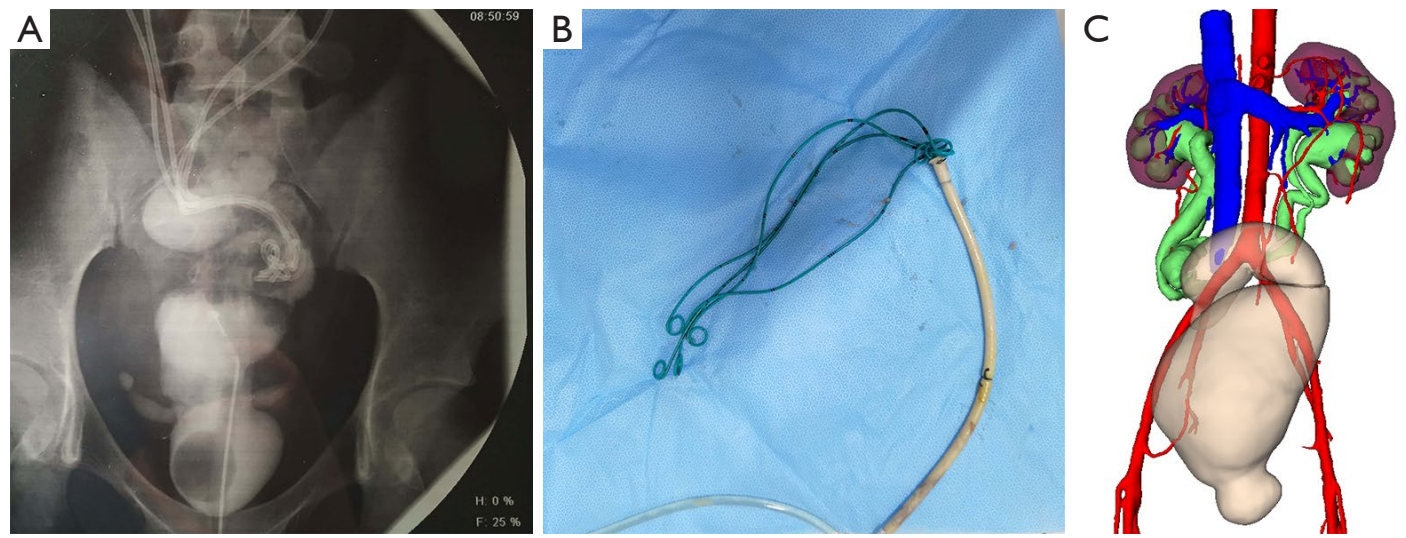

Figure 4 Postoperative management and imaging examinations. (A) Postoperative evaluation by cystography; (B) removal of cystostomy tube combined with 4 single-J stents; (C) postoperative evaluation by three-dimensional (3D) virtualization technology of computed tomography urography (CTU).

Ultrasound revealed no significant hydronephrosis and CTU with 3D virtualization technology showed no signs of stricture or obstruction. Cine magnetic resonance urography (Figure $4 C$ ) showed peristaltic motility of the bilateral duplex ureter and moderate compliance of the augmented bladder (11). The serum creatinine was $1.41 \mathrm{mg} / \mathrm{dL}$ (normal range, $0.498-1.505 \mathrm{mg} / \mathrm{dL}$ ) and the volume of the neobladder increased to $480 \mathrm{~mL}$ at 13 months postoperatively. The volume of the neobladder increased to $468 \mathrm{~mL} 6$ months postoperatively. In addition, the peak flow rate was $17.2 \mathrm{~mL} / \mathrm{s} 13$ months after surgery.

\section{Discussion}

The involvement of the tubercular urinary bladder may cause the bladder to become irregular, shrink, contract, or even calcify, which may eventually lead to auto-cystectomy (12). Augmentation cystoplasty with detubularized bowel segment is performed for patients with tubercular bladder to relieve the patient's symptoms due to a reduced bladder volume and to prevent renal damage (13). At least 3 to 6 weeks of ATT is indicated before any reconstructive surgery to relieve the inflammatory process, stabilize the disease, and for better evaluation when performing the procedure (14). Recently, orthotopic neobladder reconstruction has been reportedly used for the tubercular contracted bladder, with good results (15-17). The purpose of the reconstructive surgery is to (I) enlarge the small bladder so that the patient can retain urine for a reasonable period (II) restore function as a high-pressure compressor during micturition and as a low-pressure reservoir (less than $30 \mathrm{~cm}$ of water) during storage, and (III) prevent incontinence and infection that may compromise the integrity of the upper urinary tract (18).

There are a variety of surgical alternatives that can be used to repair the refluxing duplex collecting system, including partial nephrectomy, ureteropyelostomy, ureteroureterostomy, reimplantation of the common ureteral sheath, and sub-ureteral injection therapy. The surgical method varies according to the anatomical status of the contralateral ureter and kidney and the renal function of the refluxing kidney. The purpose of the surgical treatment is to protect renal function by preventing urinary tract infections and relieving obstruction or to achieve urinary continence in the ectopic insertion of the ureter $(19,20)$.

Our case was unique as it involved a tuberculous contracted bladder combined with a bilateral duplex collecting system. No such report has been previously published. In addition, we performed robot-assisted IUPU orthotopic ileal neobladder reconstruction for the benign disease. In our case, the patient had tuberculous bladder contracture with a history of pulmonary $\mathrm{TB}$ without renal TB. Considering the moderate renal function, bilateral duplication of the collecting system, ectopic ureteral orifice, and high-grade VUR, we decided to perform the modified and special urinary tract reconstruction to protect renal function as much as possible.

The reconstruction procedures included resection of the end of ureters and the diseased contracted bladder with preservation of the proximal urethra and bladder neck, an IUPU strategy to reconstruct a neobladder, duplex ureters tailoring, uretero-ileal anastomosis, and neobladder-bladder 
neck anastomosis. The purpose was to increase bladder capacity, decrease bladder pressure, protect the upper urinary tract, and acquire continence.

Although augmentation ileocystoplasty is the accepted treatment for tubercular bladder contracture, this may not alleviate the lower urinary tract symptoms in markedly contracted bladders, especially for suprapubic pain. The anastomoses between the reconstructive pouch and the end-stage tuberculous bladder with severe scarring may lead to anastomotic stricture and diverticulization of the pouch segment (17). Orthotopic ileal neobladder reconstruction is a viable option for tubercular contracted bladder with a markedly reduced volume (as little as $15 \mathrm{~mL}$ ) where augmentation alone might be related to poor relief of symptoms or anastomotic stricture. The procedure eliminates the symptoms, allows anastomosis with healthy tissue of the bladder neck or proximal urethra, and solves the pathological problems and VUR of the lower ureter. It also avoids the possibility of hourglass contracture, diverticularization, and spontaneous rupture. Innervation of the support mechanism of the urethra is preserved and the risk of incontinence is reduced by being close to the bladder surface (13).

To simplify the surgical procedures of pouch reconstruction and reduce the use of the ileum, we used the IUPU orthotopic ileal neobladder. The ileum has better compliance and less contractibility compared with the cecum and colon (21). Therefore, a neobladder made of ileum has better continence rates and lower pressure. Based on our initial experience, we considered several factors regarding our IUPU neobladder reconstruction technique. First, the IUPU neobladder consisted of an afferent limb and a spherical pouch which was reconstructed of detubularized ileum. According to Hinman's theory, ileal detubularization could eliminate the pressure wave caused by peristalsis and obtain minimal surface area and maximal volume from a certain ileal length (22). Furthermore, a high-capacity and low-pressure pouch could be conducive to the duplex collecting system and the ideal surface-tovolume ratio might reduce metabolic complications (23). Second, the IUPU neobladder technique is simple and quick, as it requires only twice-folding of the ileum and only two pairs of parallel edges of the ileum segment need to be sutured. Thus, the IUPU neobladder could also be appropriate for intracorporeal reconstruction with its simple sutures. Third, during the urine storage phase, the IUPU neobladder increases bladder compliance and lowers intravesical pressure. In most cases, reflux improves after augmentation, thus eliminating the need for antireflux procedures (24). With the afferent isoperistaltic ileum segment for avoiding reflux, there is no need to make another anti-reflux procedure which might prolong the operative time and increase the operation difficulty. Additionally, the robotic technique has advantages of tremor filtration, motion scaling, wrist-like instrumentation, and provides a greater degree of instrument freedom, and thus has the advantages of suturing during the entero-cystoplasty procedure $(25,26)$.

The limitations of the study are that is a single case and has a retrospective nature. The study also does not compare the IUPU neobladder to the traditional procedures. We aimed to present our robotic technique of the IUPU orthotopic ileal neobladder reconstruction for tuberculous bladder contracture. In conclusion, further studies with larger patient cohorts and a longer follow-up time are needed to evaluate the outcomes of this technique.

\section{Conclusions}

Robotic construction of the IUPU neobladder can effectively increase bladder capacity, reduce intravesical pressure, and improve symptoms, such as frequent urination and urgency caused by bladder contracture and duplex ureters. The technique of the IUPU neobladder is easy to perform and leads to few postoperative complications. However, these procedures require rigorous long-term follow-up of renal function, electrolyte levels, urinary infection, TB relapse, recurrence of hydronephrosis, and residual urine.

\section{Acknowledgments}

The authors thank the entire staff of the Department of Urology, Peking University First Hospital.

Funding: None.

\section{Footnote}

Reporting Checklist: The authors have completed the CARE reporting checklist. Available at https://dx.doi. org/10.21037/tau-21-535

Peer Review File: Available at https://dx.doi.org/10.21037/ tau-21-535

Conflicts of Interest: All authors have completed the ICMJE 
uniform disclosure form (available at https://dx.doi. org/10.21037/tau-21-535). Drs. XL and LZ currently serve as unpaid editorial board members of Translational Andrology and Urology. The other authors have no conflicts of interest to declare.

Ethical Statement: The authors are accountable for all aspects of the work in ensuring that questions related to the accuracy or integrity of any part of the work are appropriately investigated and resolved. All procedures performed in this study were in accordance with the Declaration of Helsinki (as revised in 2013) and approved by the Ethics Committee of Peking University First Hospital (approval number: 2020-283). Written informed consent was obtained from the patient for publication of this case report and accompanying images. A copy of the written consent is available for review by the editorial office of this journal.

Open Access Statement: This is an Open Access article distributed in accordance with the Creative Commons Attribution-NonCommercial-NoDerivs 4.0 International License (CC BY-NC-ND 4.0), which permits the noncommercial replication and distribution of the article with the strict proviso that no changes or edits are made and the original work is properly cited (including links to both the formal publication through the relevant DOI and the license). See: https://creativecommons.org/licenses/by-nc-nd/4.0/.

\section{References}

1. Organization WH. Global tuberculosis report 2013. World Health Organization, 2013.

2. Cek M, Lenk S, Naber KG, et al. EAU guidelines for the management of genitourinary tuberculosis. Eur Urol 2005;48:353-62.

3. Gupta NP, Kumar R, Mundada OP, et al. Reconstructive surgery for the management of genitourinary tuberculosis: a single center experience. J Urol 2006;175:2150-4; discussion 2154.

4. Hunziker M, Kutasy B, D'Asta F, et al. Urinary tract anomalies associated with high grade primary vesicoureteral reflux. Pediatr Surg Int 2012;28:201-4.

5. Davda S, Vohra A. Adult duplex kidneys: an important differential diagnosis in patients with abdominal cysts. JRSM Short Rep 2013;4:13.

6. Varlatzidou A, Zarokosta M, Nikou E, et al. Complete unilateral ureteral duplication encountered during intersphincteric resection for low rectal cancer. J Surg Case Rep 2018;2018:rjy266.

7. Privett JT, Jeans WD, Roylance J. The incidence and importance of renal duplication. Clin Radiol 1976;27:521-30.

8. Berrocal T, López-Pereira P, Arjonilla A, et al. Anomalies of the distal ureter, bladder, and urethra in children: embryologic, radiologic, and pathologic features. Radiographics 2002;22:1139-64.

9. Hong P, Ding GP, Hao H, et al. Laparoscopic Radical Cystectomy With Extracorporeal Neobladder: Our Initial Experience. Urology 2019;124:286-91.

10. Clavien PA, Barkun J, de Oliveira ML, et al. The ClavienDindo classification of surgical complications: five-year experience. Ann Surg 2009;250:187-96.

11. Zhu WJ, Ma MM, Zheng MM, et al. Cine magnetic resonance urography for postoperative evaluation of reconstructive urinary tract after ileal ureter substitution: initial experience. Clin Radiol 2020;75:480.e1-480.e9.

12. Das P, Ahuja A, Gupta SD. Incidence, etiopathogenesis and pathological aspects of genitourinary tuberculosis in India: A journey revisited. Indian J Urol 2008;24:356-61.

13. Gupta NP, Kumar A, Sharma S. Reconstructive bladder surgery in genitourinary tuberculosis. Indian J Urol 2008;24:382-7.

14. Ghoneim IA, Rabets JC, Mawhorter SD. Tuberculosis and Other Opportunistic Infections of the Genitourinary System. In: Wein AJ, Kavoussi LR, Campbell MF. editors. Campbell-Walsh urology. Philadelphia, PA: Elsevier Saunders, 2012.

15. Mochalova TP, Starikov IY. Reconstructive surgery for treatment of urogenital tuberculosis: 30 years of observation. World J Surg 1997;21:511-5.

16. Aswathaman K, Devasia A. Thimble bladder. ANZ J Surg 2008;78:1049.

17. Hemal AK, Aron M. Orthotopic neobladder in management of tubercular thimble bladders: initial experience and longterm results. Urology 1999;53:298-301.

18. Singh V, Sinha RJ, Sankhwar SN, et al. Reconstructive surgery for tuberculous contracted bladder: experience of a center in northern India. Int Urol Nephrol 2011;43:423-30.

19. Ellsworth PI, Lim DJ, Walker RD, et al. Common sheath reimplantation yields excellent results in the treatment of vesicoureteral reflux in duplicated collecting systems. J Urol 1996;155:1407-9.

20. Gerwinn T, Gnannt R, Weber DM, et al. Laparoscopic Ureteroureterostomy vs. Common Sheath Ureteral 
Reimplantation in Children With Duplex Kidney Anomalies. Front Pediatr 2021;9:637544.

21. Schrier BP, Laguna MP, van der Pal F, et al. Comparison of orthotopic sigmoid and ileal neobladders: continence and urodynamic parameters. Eur Urol 2005;47:679-85.

22. Hinman F Jr. Selection of intestinal segments for bladder substitution: physical and physiological characteristics. J Urol 1988;139:519-23.

23. Mills RD, Studer UE. Metabolic consequences of continent urinary diversion. J Urol 1999;161:1057-66.

Cite this article as: Zhu W, Li Z, Fan S, Wang X, Yang K, Xiong G, Li X, Zhou L. Management of tuberculouscontracted bladder with bilateral duplex collecting system: a case report with modified robotic urinary tract reconstructive surgery. Transl Androl Urol 2021;10(10):3891-3898. doi: 10.21037/ tau-21-535
24. Rushton HG, Woodard JR, Parrott TS, et al. Delayed bladder rupture after augmentation enterocystoplasty. J Urol 1988;140:344-6.

25. Dogra PN, Regmi SK, Singh P, et al. Robot-assisted laparoscopic augmentation ileocystoplasty in a tubercular bladder. Urol Ann 2014;6:152-5.

26. Camarillo DB, Krummel TM, Salisbury JK Jr. Robotic technology in surgery: past, present, and future. Am J Surg 2004;188:2S-15S. 